\title{
Highly electronegative contacts to compound semiconductors ${ }^{\text {a) }}$
}

\author{
R. A. Scranton, J. S. Best, ${ }^{\text {b) }}$ and J. O. McCaldin \\ California Institute of Technology, Pasadena, California 91125
}

(Received 10 February 1977; accepted 18 April 1977)

Gold contacts to most III-V and II-VI compounds position the Fermi level at the interface well into the energy gaps of the semiconductors. To position the Fermi level closer to a conduction-band edge, particularly in the more ionic semiconductors, one may substitute a more electropositive element like $\mathrm{Al}$ for the Au contact. To position the Fermi level closer to a valence-band edge, however, there are no further possibilities among the elemental metals, since $\mathrm{Au}$ is the most electronegative of these. Two contact materials, $(\mathrm{SN})_{x}$ and $\mathrm{HgSe}$, which overcome this limitation have recently been reported. Barriers produced by these contacts on many compound semiconductors will be reported and shown to exhibit the well-known ionic-covalent transition. Device use and suggestions for further research are mentioned.

PACS numbers: $73.30 .+y, 85.30 . \mathrm{Hi}$

\section{INTRODUCTION}

Metallic contacts for compound semiconductors have recently been reported which are in effect more electronegative than Au contacts. The new contact materials are potentially useful where one desires a wider range of Schottky barrier heights on compound semiconductors than that available from the present repertoire of contact materials. Recent developments in the new contacts will be reported in this paper. First, however, we briefly review the range of Schottky barrier heights available on $n$ - and $p$-type semiconductors.

Discussions ${ }^{1}$ of barrier heights often report only the barrier on $n$-type semiconductor, $\phi_{n}$. Often one must deal with $\phi_{p}$ as well, however, particularly where ohmic contact to $p$-type semiconductors is important. The two barriers are related in a simple way as may be seen in the band diagram for a rectifying metal contact, shown in Fig. 1. The barrier for holes, $\phi_{p}$, is the potential difference between the semiconductor valence-band maximum at the interface and the conductor Fermi level as depicted in Fig. $\mathbf{l}(\mathbf{b})$. It is independent of semiconductor doping as can be seen by supposing that the Fermi level in the bulk of the semiconductor is brought closer to the conduction band, as in Fig. 1(c). The barrier height for electrons, $\phi_{n}$, is the potential difference between the conduction-band minimum at the interface and the conductor Fermi level. Note that at the interface, the positions of the conduction-band minimum, the conductor Fermi level, and valence-band maximum do not change as the Fermi level in the bulk of the semiconductor is varied. In other words, $\phi_{n}$ $+\phi_{p}=E_{G}$. A metal which produces a very low barrier, i.e., ohmic contact to, say, $n$-InP, will produce a high barrier to $p$-InP.

Consider the range of barrier heights available with elemental metals. Figure 2 compares the $\mathrm{Al} / n-\mathrm{ZnS}$ barrier with the $\mathrm{Au} / \boldsymbol{n}-\mathrm{ZnS}$ barrier. Au produces higher barriers to $n-\mathrm{ZnS}$ than Al. In general, Au produces higher barriers to $n$-type semiconductors than Al. Actually, $\mathrm{Au}$ and $\mathrm{Al}$ represent the extremes of barrier height available using the common, nonreactive elemental metals. The barrier heights for other commonly used metals lie between the barrier heights for $\mathrm{Au}$ and $\mathrm{Al}$.

Over ten years ago, Mead and co-workers ${ }^{2}$ demonstrated that on many semiconductors, the barrier heights of the common metals can be ranked, or ordered, by the Pauling electronegativity scale. Figure 3 shows the Pauling electronegativity scale ${ }^{3}$ and a number of representative elements. To the right are the very electronegative elements. Electronegative conductors tend to produce high barriers to $n$-type semiconductors. The commonly used elemental metals occupy a short range on the electronegativity scale, from $\mathrm{Al}$ at 1.5 to Au at 2.4. Actually, there are a number of more electropositive metals which produce lower barriers to $n$-type semiconductors than the commonly used metals; unfortunately, these metals are very reactive. Of all the elemental metals, $\mathrm{Au}$, the most electronegative metal, produces the highest barrier to $n$-type semiconductors.

\section{ELECTRONEGATIVE METALLIC CONTACTS}

In many cases, we would like to produce contacts that are even more electronegative than Au. There are only eight elements more electronegative than $\mathrm{Au}$; not one is a conductor. In order to find a more electronegative contact we have to consider compounds. Figure 3 schematically indicates that metallic compounds may extend the range of barrier heights.

\section{A. Polymeric sulfur nitride}

Sulfur and nitrogen are very electronegative insulators. Yet a binary compound of these elements, polymeric sulfur nitride, $(\mathrm{SN})_{x}$, is a conductor. $(\mathrm{SN})_{x}$ is composed of long chains of alternating sulfur and nitrogen atoms, and has a gold brown, metallic luster. It can be grown as a crystal or deposited as a thin film. ${ }^{4}$ An important question arises: Is $(\mathrm{SN})_{x}$ more electronegative than $\mathrm{Au}$, i.e., will it produce higher barriers to $n$-type semiconductors than $\mathrm{Au}$ ? If so, then compounds can indeed extend the range of barrier heights beyond that afforded by the elemental metals. 
(a)
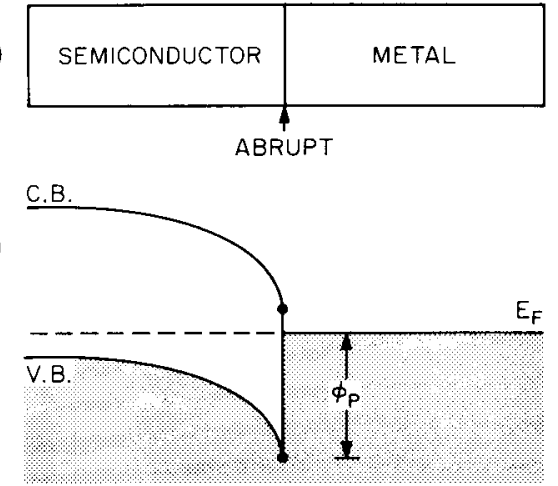

(c)

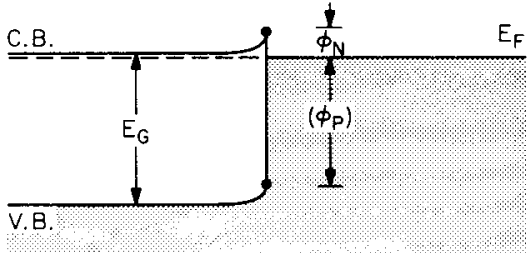

(d) $\phi_{N}+\phi_{P}=E_{G}$

FIG. 1. At intimate conductor-semiconductor contacts (a), the positions of the conduction-band minimums, conductor Fermi level, and valence-band maximum are independent of semiconductor doping. Thus $\phi_{p}$, the barrier on $p$-type semiconductors (b), plus $\phi_{N}$, the barrier on $n$-type semiconductors (c), sum to $E_{G}$, the band-gap energy.

As reported in our recent Applied Physics Letter, ${ }^{5}(\mathrm{SN})_{x}$ produces barriers that are about $1 \mathrm{eV}$ higher than barriers formed by Au on $n$-type $\mathrm{ZnS}$. Knowing this, one would expect $(\mathrm{SN})_{x}$ to produce smaller barriers or perhaps ohmics to $p$-type semiconductors. We undertook a study of the barrier heights of $(\mathrm{SN})_{x}$ and $\mathrm{Au}$ on nine different compound semiconductors.

Barrier heights for Au contacts to air-cleaved semiconductors were compared to barrier heights for $(\mathrm{SN})_{x}$ contacts. By three methods of barrier height measurement, $(\mathrm{SN})_{x}$ was shown to produce more electronegative contacts than $\mathrm{Au}$. Table I shows the difference between the barrier heights for $(\mathrm{SN})_{x}$ and for $\mathrm{Au}$. For $p$-type semiconductors, $(\mathrm{SN})_{x}$ produces lower barriers than $\mathrm{Au}$. Sometimes the resulting ohmic contact prevented determination of the barrier height at room temperature. For $n$-type semiconductors, $(\mathrm{SN})_{x}$ produces higher barriers than $\mathrm{Au}$. The improvement is not as great for covalent semiconductors as for ionic $\mathrm{ZnS}$, but $0.1-0.3 \mathrm{eV}$ is still substantial.
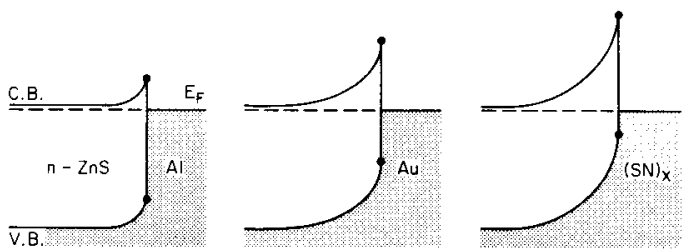

FIG. 2. The range of barriers on $n-\mathrm{ZnS}$. The barrier heights of $\mathrm{Al}$ and $\mathrm{Au}$ represent the extremes of behavior of common elemental metal contacts. Of all the elemental metals, $\mathrm{Au}$, the most electronegative, produces the highest barriers to $n$-type semiconductors. However, the binary compound $(\mathrm{SN})_{x}$, a metallic conductor, produces even higher barriers to $n$-type semiconductors than does Au.

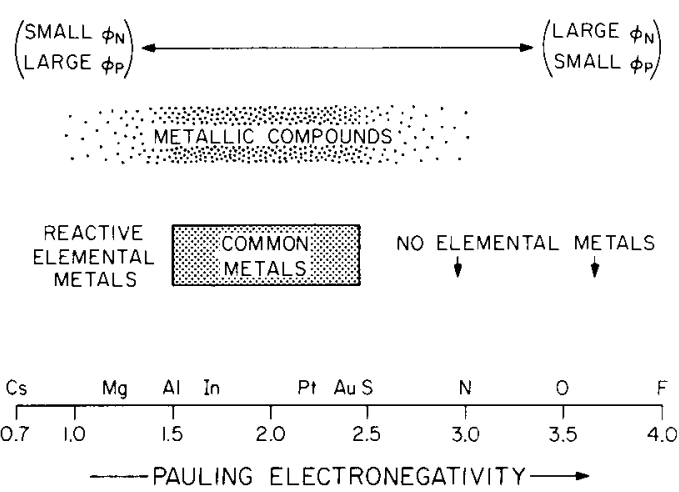

FIG. 3. The heights of barriers produced by the elemental metals can be ranked by the electronegativity of the metal. At the bottom are the Pauling electronegativity scale and some representative elements. The common metals occupy a relatively short range of the electronegativity scale and compound metals a somewhat larger range.

The barrier height improvement afforded by $(\mathrm{SN})_{x}$ is placed in perspective in Fig. 4. At the left is a reproduction of a Au barrier height compilation by McCaldin, McGill, and Mead. ${ }^{7,8}$ Briefly, it is a plot of the conduction-band minimum and valence-band maximum of the common III-V and II-VI semiconductors, using the Fermi level of $\mathrm{Au}$, in a Schottky barrier structure, as a reference level. For example, the barrier height for $\mathrm{Au}$ on $n-\mathrm{ZnS}$ is $2.0 \mathrm{eV}$ and the barrier height for $\mathrm{Au}$ on $p$-type phosphides and tellurides is about $0.8 \mathrm{eV}$. The data points represent a variety of investigators, surface preparation techniques, and measurement techniques. The important observation is that the barrier height for holes, $\phi_{p}$, depends only on the anion of the semiconductor. On the extreme right is an electronegativity scale of the elements.

This diagram can be used to estimate the barrier heights of other metals on these semiconductors. For example, the Fermi level of $\mathrm{Al}$, at an interface with $\mathrm{ZnS}$, would lie at approximately the position indicated by the electronegativity

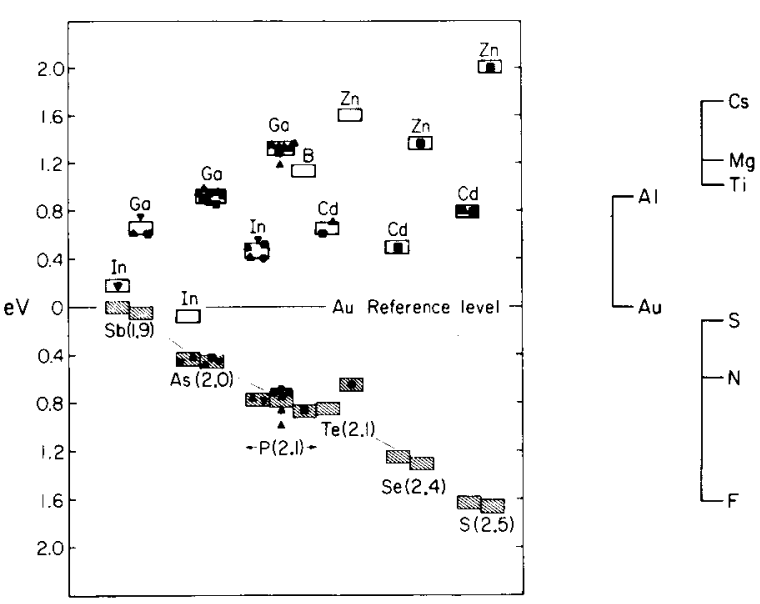

FIG. 4. At the left, the positions of the valence-band maximums (hatched rectangles) and the conduction-band minimums (shaded rectangles) of each semiconductor at an interface with Au are plotted using the Fermi level of $\mathrm{Au}$ as a reference level (after McCaldin, McGill, and Mead, Refs: 7, 8). The numbers in parentheses are the electronegativity of the anion of the semiconductor. To the right is an electronegativity scale of the elements. As described in the text, this diagram can be used to estimate the barrier heights of elemental metals on common compound semiconductors. The Fermi levels of $(\mathrm{SN})_{x}$ and the mercury chalcogenides lie below the Au reference level. 
TABLE I. Comparison of Au and (SN) $)_{x}$ Schottky barrier heights (in $\mathrm{eV}$ ) on semiconductors cleaved in air

\begin{tabular}{cccccccccc}
\hline \hline Semiconductor & $n$-GaAs & $p$-GaAs & $n$-InP & $p$-Gap & $p$-ZnTe & $n$-CdSe & $n$-ZnSe & $n$-CdS & $n$-ZnS \\
\hline$\phi(\mathrm{SN})_{x}-\phi(\mathrm{Au})$ & 0.1 & -0.1 & 0.3 & -0.2 & -0.3 & $0.1-0.2$ & $0.3-0.6$ & 0.3 & $0.9-1.0$ \\
\hline \hline
\end{tabular}

scale. The Fermi level of $\mathrm{Al}$, at an interface with one of the more covalent semiconductors (to the left side of the diagram), would lie appreciably closer to the Au reference level, however. The Fermi levels of the other commonly used elemental metals would lie between the Fermi levels of $\mathrm{Al}$ and $\mathrm{Au}$.

Notice that the Fermi level of the elemental metals, at the interface with many of these III-V and II-VI semiconductors, lies in the upper portion of the energy gaps. However, the lower portion of the energy gaps, below the Au reference level, cannot be contacted by the elemental metals. On this diagram, the Fermi level of $(\mathrm{SN})_{x}$ lies below that of Au, providing contact closer to the valence-band maximums of these semiconductors.

As mentioned earlier, very electronegative conductors can be used to facilitate ohmic contacts to $p$-type semiconductors, since one of the ways to make an ohmic is to choose a conductor which produces a very low barrier height. Ohmics to $n$-CdS, $n$-CdSe, $n$-CdTe, and $n$-InP can be made with an electropositive elemental metal such as $\mathrm{Al}$ or In. The resulting small barrier produces an ohmic contact. On the other hand, the valence-band maxima of the phosphides and tellurides lie well below the Au reference level. All the elemental metals produce relatively high barriers to the $p$-type phosphides and the tellurides. A very electronegative metallic compound would produce lower barriers to $p$-type semiconductors, thereby facilitating ohmic contacts to these semiconductors.

\section{B. Hg chalcogenides}

We will now explain the reasoning which led to a second group of very electronegative contacts. These are the mercury chalcogenides. Chalcogen refers to group VI elements: tellurium, selenium, and sulfur. Consider, for example, the selenides: The valence-band positions are anion derived and lie at the same energy with respect to the Au reference level, as illustrated in Fig. 5.

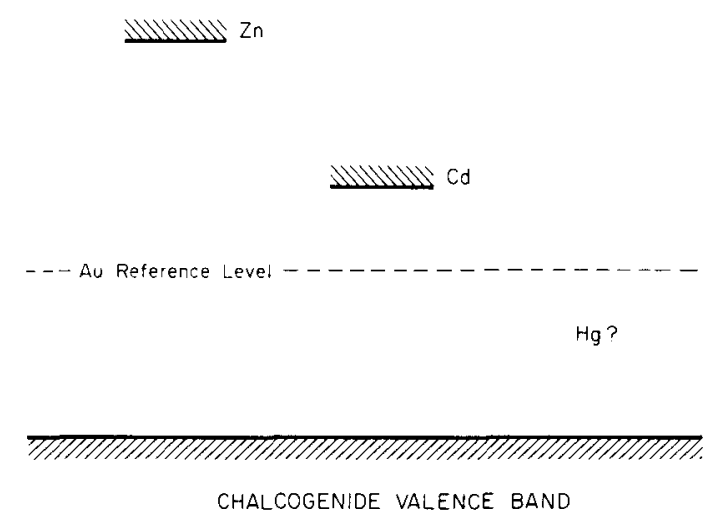

Fig. 5. A simplicication of Fig. 4 for just the II-VI semiconductors. All of the tetrahedrally coordinated $\mathrm{Hg}$ chalcogenides are semimetals with zero band gap. This diagram suggests that the conduction-band minimum of a $\mathrm{Hg}$ chalcogenide would lie below the Au reference level, thereby affording contact closer to the valence bands of common semiconductors.
As the cation changes from $\mathrm{Zn}$ to $\mathrm{Cd}$, the valence-band position remains the same. The value of the energy gap of the semiconductor determines the position of the conductionband minimum. The question arises: Where does the tetrahedral compound of the next heavier group II element, $\mathrm{Hg}$, lie on this diagram? All of the tetrahedrally bonded mercury chalocogenides are semimetals with essentially zero band gap. Depending on whether anion control of the valence-band position persists as the band gap shrinks to zero, one could expect the Fermi level of the mercury chalcogenide semimetal to lie significantly below the Au reference level. Indeed, as recently reported by Best and co-workers ${ }^{9}$ at our laboratories, $\mathrm{HgSe}$ is more electronegative than $\mathrm{Au}$, producing higher barriers to $n$-type semiconductors and lower barriers to $p$-type semiconductors than the elemental metals. Figure 6 shows the difference between the barrier heights for Au and for HgSe on some compound semiconductors. The shaded regions indicate a generous estimate of the error involved in the measurements. Compared to $(\mathrm{SN})_{x}$, HgSe offers advantages of increased stability and compatibility with semiconductor device processing.

Since the $\mathrm{Hg}$ chalcogenides may be technologically important, we will discuss the deposition of HgSe thin films. A growth chamber, made of stainless steel and quartz, was mounted inside a vacuum system as shown in Fig. 7. The chamber contains a masked sample and a drop of triple-distilled $\mathrm{Hg}$. The equilibrium vapor pressure of $\mathrm{Hg}$ at room temperature is quite high, about $1.87 \times 10^{-1} \mathrm{~Pa}\left(1.4 \times 10^{-3}\right.$ Torr). This chamber is pumped by a vacuum system, so that the $\mathrm{Hg}$ pressure within the chamber is less than the equilibrium value and $\mathrm{Hg}$ does not condense on the sample. Se is evaporated through the hole in the base onto the sample. The condensing Se reacts with the $\mathrm{Hg}$ vapor, at room temperature, to form $\mathrm{HgSe}$.

Selenium vapor can exist as $\mathrm{Se}_{2}$ or as ring structures with 3-8 atoms per molecule. At room temperature, the single bonded $\mathrm{Se}_{5}$ and $\mathrm{Se}_{6}$ ring structures are the most stable and are not very reactive. ${ }^{10}$ Figure 8 , from data compiled by Ber-

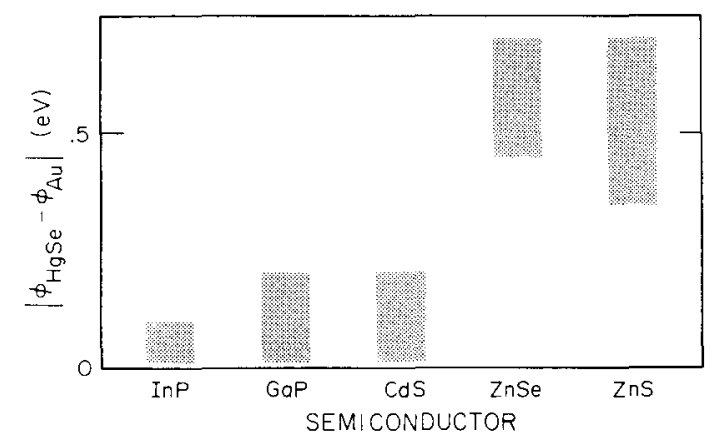

FIG. 6. The difference in barrier heights for Au and for HgSe on common compound semiconductors. The shaded regions indicate the extremes of uncertainty of the barrier height measurements. HgSe produces higher barriers to $n$-type semiconductors and lower barriers to $p$-type semiconductors than any elemental metal. 


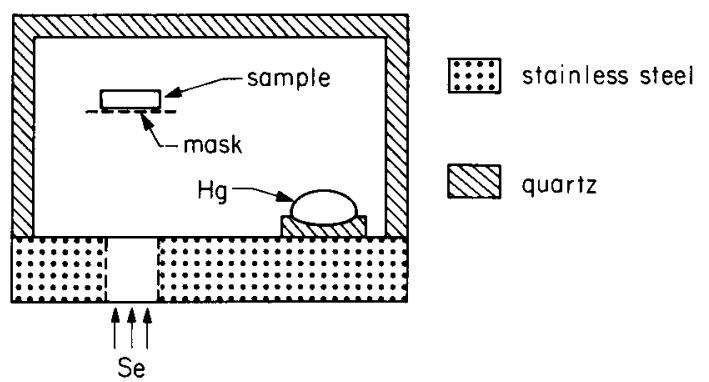

FIG. 7. An apparatus to vacuum deposit HgSe thin films: Selenium vapor condenses on the sample and then reacts with $\mathrm{Hg}$ vapor at room temperature to produce $\mathrm{HgSe}$ (reproduced from Ref. 9).

kowitz, ${ }^{11}$ shows the average number of Se atoms per selenium-vapor molecule as a function of pressure, with temperature as a parameter. Vacuum system pressures are quite low; on this scale, they are essentially zero. Se has a very high evaporation rate at $250^{\circ} \mathrm{C}$. If $\mathrm{Se}$ is evaporated from an ordinary boat or shielded source, a large proportion of the Se vapor consists of the stable ring structures. As the temperature of the vapor increases, the Se rings apparently break into more reactive diatomic $\mathrm{Se}_{2}$ molecules.

In our system, the $\mathrm{Se}$ is heated in a boron nitride crucible to perhaps $250^{\circ} \mathrm{C}$, which produces an adequate evaporation rate (see Fig. 9). The selenium vapor then passes through a very hot quartz tube, where the Se ring structures in the vapor are cracked into smaller molecules. The coiled tube has a length to diameter ratio of over 100 and the heater is a tungsten wire wrapped around the tube. $\mathrm{Se}_{2}$ vapor coming out of the top of the tube is directed into the $\mathrm{HgSe}$ growth chamber shown previously.

\section{DISCUSSION}

The use of metallic compounds, rather than just elemental metals, extends the range of barrier heights available on common semiconductors. The very electronegative conductors, such as $(\mathrm{SN})_{x}$ and the $\mathrm{Hg}$ chalcogenides, produce contacts closer to the valence band than the elemental metals.

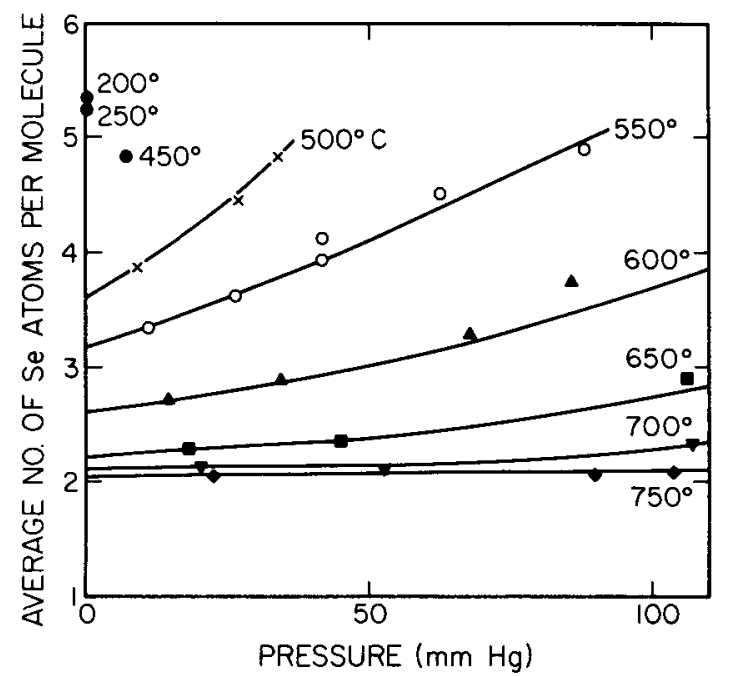

FIG. 8. The average number of Se atoms in a molecule of Se vapor is plotted against pressure, with temperature as a parameter. Heating the Se vapor changes the stable ring structure molecules to the more reactive diatomic Se $_{2}$. (From data compiled by Berkowitz and Chupka, Ref. 11).

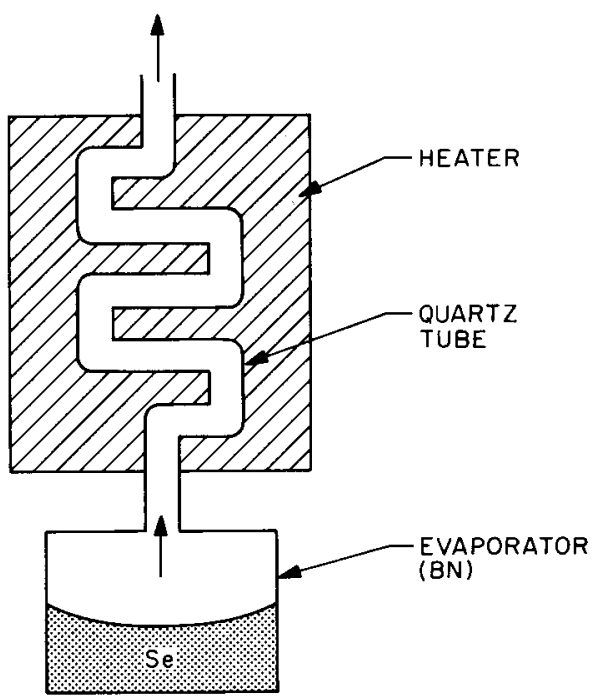

FIG. 9. An apparatus to produce reactive $\mathrm{Se}_{2}$ vapor. The temperature of the evaporator crucible determines the Se evaporation rate and the temperature of the coiled quartz tube adjusts the molecular weight of the Se vapor.

\section{A. Ionic-covalent transition}

For both $\mathrm{HgSe}$ and $(\mathrm{SN})_{x}$, the increased ranges of available barrier heights are much greater on ionic semiconductors than on covalent semiconductors. For example, on $n$ - $\mathrm{ZnS}$, the barrier formed by $(\mathrm{SN})_{x}$ is about $\mathrm{l} \mathrm{eV}$ higher than the barrier formed by Au. On more covalent $n$-type semiconductors, the barrier formed by $(\mathrm{SN})_{x}$ is only about $0.1-0.3 \mathrm{eV}$ higher than the barrier formed by Au. Similarly, the difference between the barrier height of $\mathrm{HgSe}$ and the barrier height of $\mathrm{Au}$, $|\phi(\mathrm{HgSe})-\phi(\mathrm{Au})|$, is much greater on $\mathrm{ZnSe}$ and $\mathrm{ZnS}$ than on covalent InP and $\mathrm{GaP}$. These results will be shown to be consistent with the behavior of elemental metal contacts to semiconductors.

The variation of barrier height with the electronegativity of the metal has been measured ${ }^{12}$ for many semiconductors. Kurtin, McGill, and Mead have shown ${ }^{12}$ that semiconductors divide into two groups: ionic, for which the barrier height is very sensitive to the electronegativity of the metal, and covalent, for which the barrier height is not very sensitive to the electronegativity of the metal. Thus the barrier differences, $|\phi(\mathrm{HgSe})-\phi(\mathrm{Au})|$ and $\left|\phi(\mathrm{SN})_{x}-\phi(\mathrm{Au})\right|$, should be much larger on ionic semiconductors than on covalent semiconductors.

\section{B. New possibilities}

The theoretical modelling of a metal-semiconductor interface is quite complex, perhaps involving surface reconstruction and defect structures. The usual methods for producing Schottky barriers probably produce surface damage. The elemental metals cannot easily be lattice matched to common semiconductors. Thus, the construction of a theoretical model to fit the experimental situation is difficult. Perhaps one can change the experimental situation to fit the theoretical models more closely. The electronegative contact material mercury chalcogenide can be lattice matched to a common semiconductor, namely, the corresponding cadmium chalcogenide. Thus the common anion system: HgS, HgSe, or $\mathrm{HgTe}$ with the corresponding cadmium chalcogenide 
semiconductor may produce a more perfect interface. This unique system is presently being investigated by J. S. Best of our laboratories.

There are several device possibilities for the electronegative contacts. Naturally, the lower barriers to $p$-type semiconductors may aid in the formation of ohmic contacts. The increased band bending produced in $n$-type semiconductors may be useful for InP Schottky barrier field-effect transistors, for Schottky barrier solar cells, and perhaps for light-emitting diodes.

\section{CONCLUSION}

Polymeric sulfur nitride and the mercury chalcogenides extend the available range of Schottky barrier heights. These metallic compounds produce higher barriers to $n$-type semiconductors and lower barriers to $p$-type semiconductors than do the elemental metals.

a) Supported in part by Office of Naval Research (D. Ferry and L. Cooper) b) National Science Foundation Graduate Fellow.

${ }^{1}$ See, for example, A. G. Milnes and D. L. Feucht, Heterojunctions and Metal-Semiconductor Junctions (Academic, New York, 1972), p. 195.

${ }^{2}$ C. A. Mead, Solid-State Electron. 9, 1023 (1966), and references contained therein.

${ }^{3} \mathrm{~L}$. Pauling, The Nature of the Chemical Bond (Cornell U., Ithaca, New York, 1960), 3rd ed, p. 93.

${ }^{4}$ A. A. Bright, Marshall J. Cohen, A. F. Garito, A. J. Heeger, C. M. Mikulski, and A. G. MacDiarmid, Appl. Phys. Lett. 26,612 (1975).

5R. A. Scranton, J. B. Mooney, J. O. McCaldin, T. C. McGill, and C. A. Mead, Appl. Phys. Lett. 29, 47 (1976)

${ }^{6}$ R. A. Scranton, (to be published).

${ }^{7}$ J. O. McCaldin, T. C. McGill, and C. A. Mead, Phys. Rev. Lett. 36, 56 (1976).

8J. O. McCaldin, T. C. McGill, and C. A. Mead, J. Vac. Sci. Technol. 13, 802 (1976).

9. S. Best, J. O. McCaldin, T. C. McGill, C. A. Mead, and J. B. Mooney, Appl. Phys. Lett. 29, 433 (1976).

${ }^{10}$ L. Pauling, General Chemistry (W. H. Freeman, San Francisco, 1970), 3rd ed., p. 216.

${ }^{11}$ J. Berkowitz and W. A. Chupka, J. Chem. Phys. 45, 4301 (1966).

${ }^{12}$ S. Kurtin, T. C. McGill, and C. A. Mead, Phys. Rev. Lett. 22, 1433 (1969). 